$\pm N=$

Global Journals In

(2)

\title{
2D Material-based Quantum Logic Gate Operating Via Self- Organization of Quantum Dots
}

\author{
By V. K. Voronov, O. V. Dudareva \& L. A. Gerashchenko
}

Irkutsk National Research Technical University

Abstract- In the paper, nanotrigger-based electronic device, capable of performing the quantum computation procedure, is described. The device represents a quantum logic gate formed from a two-dimensional material and controlled by a quantum dot. The operation of the quantum dot is analyzed. In the model representation, the transition between two states of the quantum dot, each of which controls the flow of the nanotransistor current (one of the shoulders of the nanotrigger), is equivalent to tunneling through an energy barrier separating the states. Fundamentally important is the fact that in one of these states the quantum dot is diamagnetic, and in the other it is paramagnetic. The paramagnetism of the quantum dot is due to the electronic exchange interaction, characteristic of the systems with unpaired electrons. Thus, the elementary self-organized 2D-material-derived logic gate disclosed in the present work can be employed for design of an electronic reversible unit. In other words, such a unit is able to prepare and to trigger the computation procedure.

Keywords: logic gate, self-organized quantum dot, nanotrigger, quantum computer, graphene.

GJSFR-A Classification: FOR Code: 020699

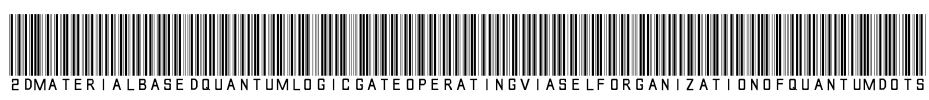

Strictly as per the compliance and regulations of:

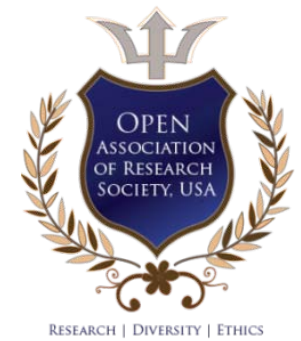

(c) 2020. V. K. Voronov, O. V. Dudareva \& L. A. Gerashchenko. This is a research/review paper, distributed under the terms of the Creative Commons Attribution-Noncommercial 3.0 Unported License http://creativecommons.org/licenses/by-nc/3.0/), permitting all non commercial use, distribution, and reproduction in any medium, provided the original work is properly cited. 


\title{
2D Material-based Quantum Logic Gate Operating Via Self-Organization of Quantum Dots
}

\author{
V. K. Voronov ${ }^{\alpha}$, O. V. Dudareva ${ }^{\sigma} \&$ L. A. Gerashchenko ${ }^{\rho}$
}

\begin{abstract}
In the paper, nanotrigger-based electronic device, capable of performing the quantum computation procedure, is described. The device represents a quantum logic gate formed from a two-dimensional material and controlled by a quantum dot. The operation of the quantum dot is analyzed. In the model representation, the transition between two states of the quantum dot, each of which controls the flow of the nanotransistor current (one of the shoulders of the nanotrigger), is equivalent to tunneling through an energy barrier separating the states. Fundamentally important is the fact that in one of these states the quantum dot is diamagnetic, and in the other it is paramagnetic. The paramagnetism of the quantum dot is due to the electronic exchange interaction, characteristic of the systems with unpaired electrons. Thus, the elementary self-organized 2Dmaterial-derived logic gate disclosed in the present work can be employed for design of an electronic reversible unit. In other words, such a unit is able to prepare and to trigger the computation procedure.
\end{abstract}

Keywords: logic gate, self-organized quantum dot, nanotrigger, quantum computer, graphene.

\section{INTRODUCTION}

T he idea of quantum computers has been first put forward by R. Feynman in his works published in the middle eighties of the twenty century $[1,2]$. The idea was based on an inference that memory and operating speed of classical computers are insufficient to solve quantum tasks. This fact can be illustrated by the following example. An $n$-particle system with two states with halfinteger spins has $2^{n}$ basic states. While solving a specific problem, $2^{n}$ amplitudes of these states should be written to the computer memory and the corresponding computations should be performed. Since $n$ can be arbitrarily large, the number of states to be operated on may also be very large. Eventually, the computations will face insuperable obstacles. Having this negative result in hand, Feynmann assumed that quantum computers would probably possess some features allowing enabling quantum problems to be solved. In addition to the aforesaid, it is pertinent to add that the creation of quantum computers is tightly connected with the problem of existence non-calculated functions and the related issue of algorithmically unsolvable tasks. These problems have been studied in

Author a $\sigma$ o: Irkutsk national research technical university, Irkutsk, Russia.e-mail:vladim.voronov1945@yandex.ru detail in the works of $\mathrm{Yu}$. Manin [3].We should also mention the papers of Paul Benioff, devoted to the behavior of quantum-mechanical objects simulating the operation of Turing machines [4-6].

It should be emphasized here that currently the application of quantum computers (if they were already created) could be effective not only for the solution of quantum physics problems. The last sixty years of the development of natural sciences were marked by appearance of new branches of knowledge, such as nonlinear optics, open system physics, and quantum information. Also, a novel area of science, which was originated at the border of several disciplines, deserves attention. We mean here the investigation of structure and dynamics of molecules. In a broad sense, this refers to the study of matter at the molecular level. The ultimate goal of such a study is electron and spatial structure of multielectron (molecular) systems as well as the nature of processes and phenomena occurring with participation of these systems. New fields of knowledge have stimulated formulation of tasks, the solution of which requires new computational devices. The problems of quantum rank high among these tasks.

Returning to the problem of quantum computer creation, it should be underlined that the most of the research performed so far involves the search for suitable individual quantum objects. At the same time it is believed that the development of the computation quantum schemes on the basis of these objects is rather a routine task. Figuratively speaking, the point is to address the issues of quantum information following the "bottom to up ideology" (see, for example, works [711] and the references cited therein). In this direction, there are some problems (in particular, the scaling and decoherence) that impede the creation of a true quantum computer. Also, one should bear in mind that the quantum computer, if it will be designed, represents a macroobject operating in a macroworld. Therefore, an alternative approach, "up to bottom", becomes possible. According to this approach, creation of devices suitable for quantum computations is based on application of initial systems of many quantum particles (available or specially obtained). Noteworthy that over the last forty fifty years, the huge amount of new compounds with the most diverse spatial and electronic structure, and, therefore, with the most various physical and chemical 
properties has been synthesized and investigated. The compounds with strong electron correlations are spectacular example.

Concluding this brief survey of the publications devoted to the problem of quantum computer creation, we can state that certain experimental advances were achieved in implementation of the ideas of R. Feynman, Yu.I. Manin, and P. Benioff. However, the design of out true quantum computer still remains an urgent challenge. As for the Orion computers of the Canadian company D-Wave is concerned, this issue requires a special discussion $[12,13]$.

Some aspects of the abovementioned "up to bottom" approach were considered in several works [14-17]. It was described in detail in a recently published paper [18], where the very idea of such an approach to solving the problem of quantum computer design was justified, and a block-scheme of electronic device, allowing this idea to be implemented, was proposed. In the present work, we extend the discussion of the device described in [18], which is really able of performing the quantum computation procedure. This material has not been published before. The authors of this work considered it appropriate to include a section with a description of the quantum computer structure at a qualitative level. Extensive literature is available on this subject[19-21].

\section{Structure of Quantum Computer: General Representation}

Schematically, the performance of a quantum computer can be represented as a sequence of three operations:

1. Recording (preparation) of the initial state.

2. Computation (unitary transformations of the initial states).

3. Output of the results (measurement, projection of the final state).

Recording of the initial state: Classic computers are based on transistor circuits having non-linear relationships between input and output voltages. Essentially, these are bistable elements. For example, at low input voltage (logic "0"), the output voltage is high (logic "1"), and vice versa. It is known that an ordinary (classical) computer operates with bits, Boolean variables that take values 0 and 1 . Moreover, at any stage of the computation, the computer contains certain values that can be measured. At the first stage of computations, one should write the initial data into the register, a set of bits, each of which must have certain values (0 or 1$)$.

Any mathematical action (multiplication, division, root extraction, etc.) ultimately reduces to addition. Therefore, the main task of the computer is addition of the numbers written in binary form. The corresponding computer element is called the adder. The latter can be built from the three simplest elements, which are connected to each other in an appropriate manner. These are logic elements or logical gates "NOT", "OR", "AND". They are called logical because they are responsible for certain logic operations (the name of each element corresponds to the performed logic function), i.e. they connect the input and output logic variables (zeros and ones). Using these three logic elements ("NOT", "OR", "AND"), it is possible to create not only an adder, but also a trigger, a decoder, a code converter and other devices that are required for a computer to function. The fact that these three elements are enough for design of almost any computer unit ultimately reflects undeniable truth: any thought can be expressed with a help of three logic connectives, "NOT", "OR", "AND". From the above gates one can go to other sets, sufficient for the manufacture of any computing device, for example, to such: "NOT", "OR-NOT", "ANDNOT".

A quantum computer operates with states. The simplest system that performs a function similar to bits in classic computers is a system with two possible states. To designate the state of such a quantum twolevel system, a special term is proposed, q-bit (quantum bit of information). It describes state of a quantum system with two possible basic states $|0\rangle$ and $|1\rangle$ The general state of such a system is a superposition:

$$
|q\rangle=C_{0}|0\rangle+C_{1}|1\rangle
$$

which is something more than boolean 0 or 1.Q-bit is a quantum superposition of two numbers: zero and one! It can therefore be said that the idea of a quantum computer is based on the fact that superposition of states is possible in quantum mechanics. A quantum system with two basis states (q-bits) allows encoding the numbers 0 and 1 in these states.

Physical systems that realize q-bits can involve any objects having two quantum states: polarization states of photons, electronic states of isolated atoms or ions, spin states of atomic nuclei, lower states in quantum dots. A full-scale quantum computer should contain a large number of q-bits (hundreds and even thousands), so that it can solve real computation problems. Therefore, the state of a quantum computer is nothing but a very complex, entangled state. Mathematically, it is described by the sum of a large and even huge number of terms. Each of these terms is a product of states of the type $|0\rangle$ or $|1\rangle$. The factors in this product describe the possible states of individual q-bits in a long chain. In other words, the state of a quantum system of $n$ two-level particles is a superposition of $2^{n}$ basic states. Eventually, the quantum principle of superposition of the states can impartfundamentally new "abilities" to a quantum computer. 
The very first operation of all (classical and quantum) computations, i.e. preparation of the initial state of the register, demonstrates the possible advantages of quantum operations with q-bits. When entering an initial number to a classical register consisting of $n$ bits, $n$ operations is required, i.e. the values 0 or 1 should be set on each bit. In this case, only one number of length $n$ will be written. When performing certain unitary operations with each q-bit, it is possible to create a coherent superposition of all $Q=$ $2^{n}$ states of the general quantum register system. Thus, instead of one number, all $2^{n}$ possible values of the register, a coherent superposition of all possible numbers for this register, can be prepared. Naturally, this property can be employed for quantum parallel computations.

It should be emphasized here that these cells are not some specific elements of scheme and not magnetic domains, onto which the necessary information is recorded. This refers to eigen values of the operator of a physical quantity characterizing the qbits system, which, according to the laws of quantum mechanics, can be determined with varying degrees of probability. In a certain sense, we are talking here about a large set of virtually availabile cells, the use of which is far from obvious. This is one of the fundamental problems. Further, after information (for example, digital) is entered into these cells, the latter should somehow be controlled to perform computational operations. Finally, one should to find a way to output the results after completion of the computation. From the above it is easy to draw a conclusion about fundamental difficulties that have to be overcome in the way of creating a quantum computer.

Computation. Applying unitary transformations that perform certain logic operations to the prepared states, it is possible to create real quantum processor. q-Bits play the role of connectors, while unitary transformations serve as logic blocks (gates) for computation both in the classical and in quantum processor. This concept of a quantum processor and quantum logic gates was proposed in 1989 by D. Deutsch, who also found a universal logic unit for performance of quantum computations. In 1995, it was shown that one- and twoq-bit gates are enough to obtain the entire necessary set of transformations.

A single q-bit element (gate) can be defined by a $2 \times 2$ matrix, which has the following form

$$
Q(\theta, \varphi)=\left(\begin{array}{cc}
\cos \frac{\theta}{2} & -i \exp (-i \varphi) \sin \frac{\theta}{2} \\
-i \exp (i \varphi) \sin \frac{\theta}{2} & \cos \frac{\theta}{2}
\end{array}\right)
$$

The gate $Q(\theta, \varphi)$ describes the rotation of the qbit state vector from the $z$ axis to the polar axis defined by angles $\theta, \varphi$. Physical implementation of this gate requires the external action a pulse on the quantum particle (q-bit) to transfer the latter from one state to another. The "OR-NOT" gate can be realized by the action of the corresponding sets of pulse sequences on two interacting $q$-bits (in particular, the spin). In this case, one q-bit should control the evolution of other. The toolkit of radio engineering and electronics has a wide range of pulsed radio-frequency sequences, which (at least, in principle) can ensure unitary transformations over a system of q-bits with a selective action on each of them.

Output of the result: In classical computer, the output of computation result does not differ from any other computational operation. The computations can be stopped at any point, the intermediate results can be read and calculations can be further continued. In a quantum computer, it is not the case. The ultimate result of quantum computation is the state of quantum register (after performance of unitary transformations), which represents a coherent superposition of all possible states for this register. Obviously, we cannot obtain all amplitudes of probability $\mathrm{C}_{j}$ in the decay of this superposition state. All that we can obtain from this single quantum object, according to the quantum theory, is quadratic forms $\sum_{i, j} C_{i} C_{j}^{*} R_{i, j}$, corresponding to measuring the average value of a certain physical quantity, which is designated by operator $\hat{R}$. It is obvious that the final result of quantum computation will change from one operation to other. However, even under such conditions of quantum uncertainties, quantum computers can significantly accelerate the computation of various mathematical problems.

The design of a quantum computer faces, first of all, physical problems. The major problem is the rapid decay of superposition states and their transformation into a mixture. This process, as noted above, is called decoherence. The latter imposes the main requirement on the physical elements that are supposed to be used in quantum computers: the time of state coherence conservation should exceed the time of computation. Hence, there are two ways of avoiding decoherence: (i) to find a quantum system that is isolated from the environment as much as possible, or (ii) to increase artificially the coherence time.

Another problem in design of quantum computers (it may be called technological)relates to the fact that after the computational process is complete, the states of q-bits should be measured to determine the results of the computation. To date, there are no reliable methods for such measurements. However, it is believed that the routes to the development of such methods are outlines: one should employ amplification procedures of quantum mechanics. For example, the state of nuclear spin / is transferred to the electronic spin 
$S$. The latter defines the orbital wave function. Having the orbital wave function in hand, the transfer of charges (ionization) can be triggered. The presence or absence of a single electron charge can be detected by classical electrodynamic methods.

\section{ili. 2D Material-Based Elementary Self- Organized Logic Gate}

In the introduction of this paper, we have mentioned that a quantum computer can be created using the "up to bottom" idea reported in [18].In that work it is proposed a block-scheme of possible electronic device, which, in principle, can help in implementation of this idea. The features of such a device are discussed below.

a) The device includes a block containing at least $10^{10}$ nanotriggers that perform a role of q-bits of quantum computation, which are formed from graphene (or other 2D material) nanoribbons and controlled by a special element. The latter represents a self-organizing quantum dot having two essentially different states in terms of magnetic properties. This quantum dot is prepared on the basis of a compound, the molecules of which are characterized by the intramolular rearrangement. The nanotriggers are employed to produce reversible logic blocks or gates. Each gate contains three triggers to perform logical operations.

b) This system of the nanotriggers correlates with all possible states of a quantum system that provide a large mathematical information resource.

c) Fundamentally important is that the quantum dot can be formed from materials based on compounds, in which intramolecular processes lead to the population of metal $3 \mathrm{~d}$-orbitals due to valence tautomerism.

d) The device represents an additional electronic unit that is embedded in a digital computer, which makes it possible to implement the computational process in accordance with the provisions of quantum physics.

The following explanation is needed here. If the proposed device is quantum in its essence, then it is obvious that its operation should obey to the laws of the microworld. In addition, the triggered device should perform certain operations (in the case of a quantum computer, these are computations) also in accordance with the laws of quantum physics. It is well known that the main elementary unit of a computer is a trigger. If it is obtained (as proposed in this paper) from twodimensional material, for example, graphene, this will mean that the first condition indicated above is fulfilled. If the computation procedure is controlled by a quantum object, which is self-organized due to intramolecular dynamics, then the above second condition can also be considered feasible.

In the model representation, the transition between two states of a quantum dot, each of which controls the current flow of the nanotransistor (one of the shoulders of the nanotrigger), is equivalent to tunneling through an energy barrier that separates these states, which differ in their magnetic properties. This process is shown in Fig. 1.

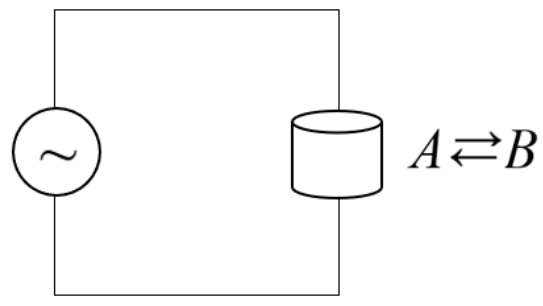

\section{Fig. 1: Operation of a control quantum dot}

Here $A$ and $B$ are the states, between which an intramolecular transition of molecules (or even clusters) occurs. A control quantum dot is formed on the basis of these states. We are talking about molecular (multielectron) systems with unpaired electrons, which properties are determined by electronic exchange interaction. It is pertinent to note here that in recent years intense research interest is focused on heterospin systems synthesized from the coordination compounds of paramagnetic transition metal ions with stable nitroxyl radicals. This is primarily due to the fact that such compounds allow obtaining detailed information on the nature of intramolecular interactions in multi-electron systems, the specificity of which is determined by the exchange magnetic interactions of unpaired electrons. Ultimately such information permits to solve fundamental problems related to the problem of the structure and dynamics of molecules. In turn, the solutions of these problems substantiate the preparation of materials with practically useful properties (see, for example, a comprehensive publication [22]).

Among fundamental processes, the intramolecular rearrangements involving delocalization of unpaired electrons from ligands to metal (e.g., cobalt) ion to change valency of the latter hold a specific place. Such rearrangements are commonly referred to as valence tautomerism. As applied to compounds of the first transition group, these intramolecular processes lead to the population of metal 3d-orbitals with unpaired electrons. The scheme given below illustrates the aforementioned rearrangement. The investigations have shown that magnetic properties of compounds can change dramatically in the course of transition of molecules from state $A$ to state $B$ and back. Fundamentally important is the fact that the molecules in state $A$ are diamagnetic, while in state $B$ they are paramagnetic. This takes places, for example, in the 
case of $\mathrm{Co}(\mathrm{SQ})_{2}\left(2-2^{\prime}\right.$-dipyridine $)$ complex, which is characterized by the above tautomerism [23] .

$\mathrm{Co}^{\mathrm{III}}(\mathrm{SQ})_{2}$ (2-2'-dipyridine) $\rightleftarrows \mathrm{Co}^{\mathrm{II}}(\mathrm{SQ})_{2}$ (2-2'-dipyridine).

\section{A $B$}

The intramolecular tautomerism can be compared with a process that ensures generation of electromagnetic oscillations at a quantum level. In this case, the periodic appearance of an unpaired electron spin on the coordination cobalt atom (which, in fact, acts as an antenna) corresponds to the radiation of the aforementioned oscillations, thus providing automatic control of the nanotrigger performance.

Fig. 2 shows a simplified standard equivalent scheme of switching on of the nanotransistor connection with a controlling quantum dot in the base circuit. The designations in this figure have generally accepted meanings.

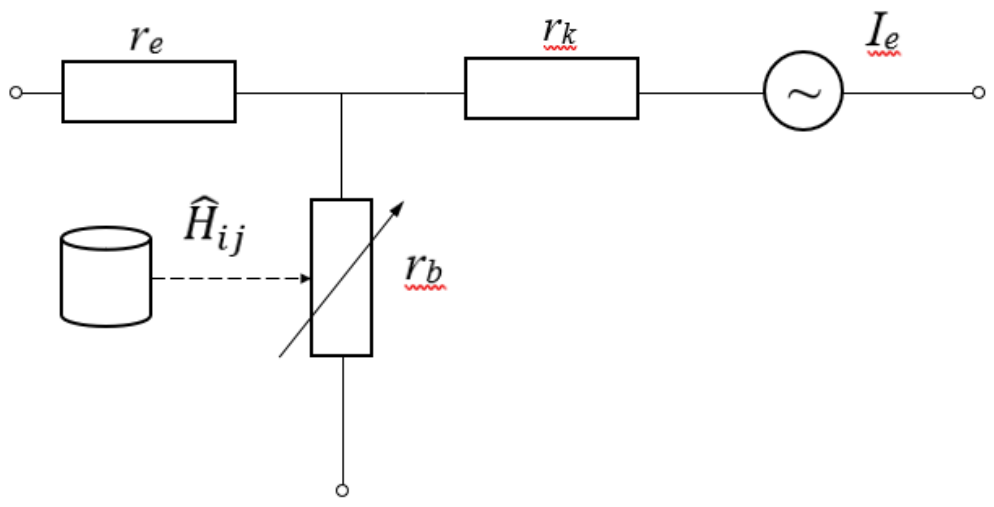

Fig. 2: A simplified equivalent scheme of switching on of nanotransistor connection with a control quantum dot in the base circuit (indices correspond emitter - e, collector - k, base - b).

A description of the performance characteristics of transistors, as well as the processes occurring in them, can be found in the corresponding books on electronics. Here, apparently, it is necessary to explain how the above quantum dot can switch on and off a transistor that reads information? At the level of the macroworld, this would naturally be done by the action of, for example, inductive coupling on the resistance of the circuit base. However, a quantum dot designed to control the operation of a nanotransistor (and the nanotrigger as a whole) is an object, which behavior is determined by the laws of quantum physics (at the microworld (evel). Therefore, a method for implementation of the action on the resistance of base should be sought starting from the above inference. Such method may comprise the exchange electron spin-spin coupling interaction, described in the Hamiltonian by $\widehat{H}_{i j} \sim J_{i} J_{k}$ term. This coupling leads, as noted above, to the generation of uncompensated electron spin on the coordination ion (in our case, on the cobalt ion). As a result, the quantum dot is magnetized for a while. This paramagnetism will change the magnetic field with a frequency corresponding to the transition between states $A$ and $B$. In this case, this corresponds to change in the magnetic permeability of the substance $\mu$. It is well known that the latter parameter directly relates to the inductance $L$, which (other conditions being equal) determines the magnitude of the inductive current in the circuit. In turn, this dependence should periodically change the current (between its maximum and minimum values) in the transistor. This is shown in Fig. 2 where the base resistance $r_{b}$ is variable.

The parameters $\mu$ and $L$ allow explaining the possible changes of $r_{b}$ by the integral action of the periodically occurring magnetization. However, if to take into consideration a deeper (molecular) level of such an action, then it is logical to use the band theory. According to this theory, the interaction described by the term of the hamiltonian $\widehat{H}_{i j}$ will periodically change width of the band gap between the states with the minimum and maximum values of the probability of electron density in the base, and, consequently, the value of potential with respect to the collector.

It is easy to conclude that in each specific case, the operating mode of an electronic device can be set by selecting the molecular structure of the substance, on the basis of which the quantum dot is synthesized. In particular, the parameter characterizing a periodically induced magnetic field can be described by an expression $B_{0} \cos \omega t\left(B_{0}\right.$ is the amplitude value of the magnetic field induced by the quantum dot). Technologically, it is not so difficult to introduce a quantum dot with the corresponding intramolecular dynamics into the transistor, given the location of the base in this transistor.

The nanotrigger can be used to perform all logical operations involved in the computational 
process. Indeed, from the perspective of radio engineering, 2D material-derived trigger, which is controlled by a self-organized quantum, represents an inverter capable of independently performing the corresponding logical operation NOT. As for the logical operation OR-NOT is concerned, it can be performed, when a quantum dot is included in each shoulder of the nanotrigger. It is well known [21] that the operation CONDITIONAL NOT ("OR-NOT")

$$
\hat{T}_{X O R}=|\mathbf{0}\rangle_{11}\left\langle\mathbf{0}\left|\hat{J}_{2}+\right| \mathbf{1}\right\rangle_{11}\langle\mathbf{1}| \hat{\boldsymbol{T}}_{2 N O T},
$$

is applied to two q-bits, the first of which does not change its state under the action of $\hat{T}_{X O R}$, and the second one changes, but depending on the state of the first q-bit. For instance,

$$
\hat{T}_{X O R}\left(\alpha|0\rangle_{1}+\beta\left\langle\left. 1\right|_{1}\right)|0\rangle_{2}=\alpha|0\rangle_{1}|0\rangle_{2}+\beta|1\rangle_{1}|1\rangle_{2},\right.
$$

i.e. the operation $\hat{T}_{X O R}$ transforms superposition states into entangled ones and vice versa.

Thus, the nanotrigger corresponds to two qbits. The load of such a trigger should be another trigger containing only one quantum dot. It should serve as the q-bit of the target.

In relation to the device described here, the first shoulder of the nanotrigger, in which the current is flowing, corresponds to the alpha state, and the second shoulder relates to the beta state. On cobalt, the spin is not equal to zero, the resistance (inductive, reactive, non-ohmic) is large, and current is absent in the circuit it is 0 . On cobalt, the spin is zero, the resitance is minimum, the current in the circuit is maximum - it is 1 (or vice versa). A device that autonomously periodically opens and closes can be compared to a self-oscillating process. Fundamental in this case is the selection the quantum dot material at the molecular (quantum) level.

The nanotrigger described above should be employed to design a reversible electronic block. In other words, such a block should prepare the computation procedure and trigger it. The continuation of the computation is the task of other computer elements. Naturally, this elementary (basic) block should operate, as already noted above, according to the laws of quantum physics. This condition imposes certain requirements on the computer architecture, including the interface used for communication with the macroworld. Such an elementary block can be compared with a generator, which once started to work, then arbitrarily performs an oscillation process. In this case, the feedback function, which is necessary to trigger the generation process), is performed by a quantum dot. The latter, due to the self-organization, reads the input information and triggers the computation (the transistor alternately changes between two states: when current is absent or present in the circuit). The output of such a transistor is connected to a block of two nano-transistors containing a quantum dot in the circuit, which are able to implement a logical operation OR-NOT. A quantum dot operates due to the energy equal to $k T$ coming from the environment (heat reservoir).

It is appropriate to note here the features of $2 \mathrm{D}$ material-based electronic device disclosed in this paper. On the one hand, it employs the classical method for fixation of the state (zero and one) by a trigger. On the other hand, the application of the nanotrigger and quantum dot as a control element ensures steady operation of such a scheme. A nanotrigger can be associated with two connected q-bits, each of which symbolizes one shoulder of the nanotrigger. Classically, the input signal is a control signal. At the same time, it is employed for communication with the macroworld (interface). In the device proposed here, such a role is played by the quantum object controlled by the quantum dot. Thus, the entire computation process is performed according to the laws of quantum physics.

Finally, it should be emphasized that the idea of design of self-organized logical gate can be fundamental and quite fruitful. In fact, instead of the quantum dot proposed in this paper, which is characterized by intramolecular processes with two substantially different states (magnetic properties), one can offer any other objects (devices included in logical blocks) of the periodic transition of the trigger shoulders to the state of zero or one. It can also be assumed that different quantum dots used in a particular electronic device depending on their (these points) functions can also be employed in the above design.

\section{Conclusions}

In the introduction, the idea of quantum computer design is briefly discussed. It is stressed that the most of the research performed so far involves the search for suitable individual quantum objects. At the same time it is believed that the development of the computation quantum schemes on the basis of these objects is rather a routine task. In other words, the point is to address the issues of quantum information following the "bottom to up ideology". In this direction, there are some problems (in particular, the scaling and decoherence) that impede the creation of a true quantum computer. Therefore, an alternative" up to bottom" approach becomes possible. According to this approach, creation of devices suitable for quantum computations is based on application of initial systems of many quantum particles (available or specially obtained). Therefore, the "top_down" approach seems to be possible. Here, the creation of devices suitable for quantum computing will be based on the use of initially systems of many quantum particles (existing or specially obtained materials). Earlier, one of the authors of this publication described a block-scheme of an electronic 
device based on a priory visualized states of q-bits. The role of the latter should be played by nanotriggers obtained from graphene or other 2D material, the performance of which is controlled by a self-organized quantum dot. In the present paper, the proposed electronic device is discussed in detail.

The operation of the quantum dot acting as a control element is described. In the model representation, the transition between two states of the quantum dot, each of which controls the flow of the nanotransistor current (one of the shoulders of the nanotrigger), is equivalent to tunneling through an energy barrier separating the states. Fundamentally important is the fact that in one of these states the quantum dot is diamagnetic, and in the other it is paramagnetic. The paramagnetism of a quantum dot is due to the electronic exchange interaction characteristic of systems with unpaired electrons. As a result, the quantum dot is magnetized for a while. This paramagnetism will change the magnetic field with a frequency corresponding to the transition between two states with different magnetic properties. In this case, this corresponds to change in the magnetic permeability of the substance $\mu$. It is well known that the latter parameter directly relates to the inductance $L$, which (other conditions being equal) determines the magnitude of the inductive current in the circuit. In turn, this dependence should periodically change the current (between its maximum and minimum values) in the circuit. The nanotrigger can be associated with two connected q-bits, each of which symbolizes one shoulder of the nanotrigger. Classically, the input signal is a control signal. At the same time, it is employed for communication with the macroworld (interface). In the device proposed here, such a role is played by the quantum object controlled by the quantum dot. Thus, the entire computation process is performed according to the laws of quantum physics.

\section{References Références Referencias}

1. Feynman R.P. Simulating Physics with Computers. International Journal of Theoretical Physics. 1982, vol. 21 (6/7), p. 467-488.

2. Feynman R.P. Quantum Mechanical Computers. Foundations of Physics. 1986,vol.16 (6), p. 507-531.

3. Manin Yu. I. Calculated and non-calculated. M.: Sovetskoye radion, 1980.-128 p.

4. Benioff P.Quantum mechanical Hamiltonian models of discrete processes that erase their own histories: Application to Turing machines. International Journal of Theoretical Physics. 1982, vol.21(3), p.177-201.

5. Benioff P. Quantum Mechanical Models of Turing Machines That Dissipate No Energy. Physical Review Letters. 1982, vol. 48(23), p.1581-1585.
6. Benioff P. Quantum mechanical Hamiltonian models of Turing machines. Journal of Statistical Physics. 1982, vol. 29(3), p.515-546.

7. Harty T. P., Sepiol M. A., Allcock D.T.C., Balance C.J., Tarlton J.E., Lucas D. M. High-Fidelity Trapped-lon Quantum Logic Using Near-Field Microwaves. Phys. Rev. Lett. 2016, vol. 117, p.140501.

8. Ryabtsevl.I., Beterov I.I., Tretyakov D.B., Entin V.M., Yakshina E.A. Spectroscopy of cold Rydberg rubidium atoms for application in quantum information. Usp. Phyz. nauk. 2016,vol. 86 (2),p.206220.

9. Gaebler J.P.,Tan T.R.,Lin Y.,Wan Y., Bowler R., Keith A.C., Glancy S., Coakley K., Knill E., Leibfried D., Wineland D.J.," High-Fidelity Universal Gate Set for ${ }^{9} \mathrm{Be}+$ Ion Qubits" Phys. Rev. Lett. 2016, vol. 117, p. 060505.

10. Usher N., Dan Browne E.Noise in one-dimensional measurement-based quantum computing. Quantum Information \& Computation.2017, vol. 17 (15-16), p.1372-1397.

11. Raedt H., Jin F., Willsch D., Willsch M., Yoshioka N., Ito N., Yuan S., Michielsen K. Massively parallel quantum computer simulator, eleven years later. Computer Physics Communications. 2019, vol. 237, p. 47-61.

12. Lanting T., Przybysz A.J., Smirnov A. Yu., Spedalieri F.M. at al. Entanglement in a Quantum Annealing Processor. Physical Review X. 2014, vol. 4, p. 021041.

13. Rønnow T.F., Wang Zh., Job J., Boixo S., Isakov S.V., Wecker D., MartinisJ.M., Lidar D.A., Troyer M. Defining and detecting quantum speedup. Science , 2014, vol. 345, p. 420-424.

14. Voronov V.K., NMR and the Problem of Quantum Computer Creation: New Outlook, in:S.Shannon (ed.), Trends in Quantum Computing Research, NOVA Publishers: New York, 2006, p. 73-90.

15. Voronov V.K.Possible schemes of calculation modeling in a quantum computer. Natural Science. 2010, vol. 2 (8), p. 923-927 (2010).

16. Voronov V.K. Physical problems of quantum calculation: A novel approach.Journal of Physical Science and Application. 2012, vol. 2 (4), p.115-122.

17. Voronov V.K. A Possibility of Application of Wave Flow Method for Visualization of Shor States.Int. J. Communications, Network and System Sciences. 2013, vol. 6, p. 485-488.

18. Voronov V.K. Quantum-dot Controlled Electronic Block Triggering a Quantum Computation Procedure.International Journal of Information Technology and Computer Science. 2020, vol.12 (2), p. $42-48$.

19. Kilin S.Ya. Quantum information. Usp. Phyz. nauk. 1999, vol.169 (5), p. 507-526. 
20. Jones J.A. NMR Quantum Computation, in Quantum Entanglement and Information Processing. D. Esteve, J.-M. Raimond, and J. Dalibard (Eds.), Elsevier Science, 2004.

21. Valiev K.A., Quantum computers and quantum computation. Usp, phyz. nauk. 2005, vol. 175 (1), p.3-39.

22. Ovcharenko V., Fursova E., Romanenko G., Eremenko I., Tretyakov E., Ikorski V.Synthesis, Structure and Magnetic Properties of (6 - 9)-Nuclear $\mathrm{Ni}(\mathrm{II})$ Trimethylacetates and Their Heterospin Complexes with Nitroxides. Inorganic Chemistry. 2006, vol. 45 (14), p.5338-5350.

23. Sato O., Tao J., Zhang Y.-Z., Control of Magnetic Properties through External Stimule. Angew. Chem. Int. Ed. 2007, vol. 46, p. 2152-2171. 